\title{
La structure des mobilités domicile-travail au départ des villes moyennes : réflexions sur leur durabilité
}

\author{
The structure of commuting from French middle-sized cities: \\ considerations on its sustainability
}

\author{
Benoit Conti \\ (C) IFSTTAR et Éditions NecPlus 2015
}

Résumé Les travaux questionnant la durabilité des déplacements pendulaires dans les différents espaces des aires urbaines, notamment en termes d'émission de polluants et de consommation énergétique, sont désormais nombreux. Ces études prennent principalement en compte les déplacements des actifs à l'intérieur des agglomérations et considèrent plus rarement la mobilité des personnes qui ont leur emploi dans une autre aire urbaine ou dans l'espace rural. La particularité de cet article est de se focaliser sur les déplacements des actifs quittant leur aire urbaine de résidence, et ce, à l'échelle des villes moyennes (aires urbaines de 100000 à 500000 habitants), moins étudiées que les grandes villes. Notre but est d'apporter une connaissance précise de ces mobilités en matière de catégories socioprofessionnelles, de distances parcourues, de modes de transport utilisés, de localisations des actifs et des emplois (ville centre, pôle urbain, espace périurbain ou rural) et de directions des flux (métropoles, ville moyennes, petites villes ou diffus), afin de nourrir une réflexion sur la durabilité environnementale de ces déplacements et les potentiels de report modal. Les résultats montrent que ces déplacements ne sont pas limités aux catégories professionnelles supérieures. Ils rendent également compte d'une forte utilisation de la voiture sur de grandes distances et de l'existence de potentiels de mobilités alternatives (autres que l'autosolisme) différenciés qui dépendent du tissu urbain du lieu d'emploi et du lieu d'habitat.

Mots clés Mobilité interurbaines $\cdot$ domicile-travail $\cdot$ ville moyenne · altermobilité

Abstract Numerous studies have focused on the sustainability of commuting in different localisations in urban

Benoit Conti $(\bowtie)$

UMR Laboratoire Ville, Mobilité, Transport (LVMT), Université Paris-Est

6-8 Avenue Blaise Pascal Champs sur Marne F-77455 Marne la

Vallée Cedex 2

e-mail : benoit.conti@enpc.fr areas, particularly in terms of GHG emissions. They mainly take into account journeys within cities and rarely consider individuals leaving them to work in another urban or rural area. The particularity of this article is to focus on medium-sized cities (French urban areas with populations between 100,000 and 500,000 inhabitants) which have been less studied by researches on commuting and on the commute of workers leaving their urban area of residence. The aim of this study is to provide detailed results of their mobilities in terms of social categories, distance travelled, modal choice, locations of workers and jobs (city center, urban center, suburbs or rural area) and directions flow (to cities, medium cities, small cities or sprawl area) in order to provide input on the sustainability of this type of commuting and on potential for modal shift. Our first results show that commuting out of one's home urban area is not limited to higher professional classes. They confirm that workers use their car for commuting on long distances and that some travel, depending on the place of employment and housing, are more easily transferable to sustainable mode of transportation than others.

Keywords Interurban mobility · commuting · middle size town $\cdot$ sustainable transport

\section{Introduction}

Les trajets domicile-travail ne représentent plus la majorité des motifs de déplacements des Français mais tiennent encore une place importante en termes de distance, de temps et de structuration des programmes d'activité des actifs en emploi [1]. La distance des trajets pendulaires tend à augmenter depuis le début des années 1970, en lien avec le développement de la périurbanisation, l'essor de l'automobile et le desserrement des emplois $[2,3]$. Les territoires situés en périphérie des grandes agglomérations portent en effet une large responsabilité dans l'allongement des navettes quotidiennes entre le lieu de résidence et le lieu d'emploi. En revanche la relation entre la forme urbaine d'une aire urbaine (monocentrique 
ou polycentrique), la place de la voiture et la distance des navettes est plus discutée [4,5,6]. La littérature sur les causes de l'allongement des distances domicile-travail et de l'usage croissant de la voiture fait une place hégémonique aux flux intra-urbains et aux grandes aires urbaines. La problématique des actifs effectuant de longues distances entre agglomérations est un processus moins connu, derrière lequel on colle l'image plutôt simple de jeunes cadres se déplaçant en TGV [7]. Plusieurs travaux récents montrent pourtant que ces déplacements, certes moins conventionnels, sont plus complexes que cela et qu'ils méritent d'être étudiés pour d'une part, mieux les connaître, et d'autre part, évaluer leur durabilité (émissions de gaz à effet de serre, vulnérabilité des liaisons effectuées et accès aux emplois notamment) $[8,9]$. Plus largement, les mobilités domicile-travail impliquant de longues distances questionnement l'articulation entre mobilité quotidienne et mobilité résidentielle : pour Kaufmann, "la classique distinction entre la mobilité quotidienne renvoyant aux déplacements réalisés au sein d'une aire urbaine dans le cadre de la vie quotidienne et la mobilité résidentielle comme un parcours se construisant au fil du cycle de vie est chahutée par le développement de formes de déplacement hybrides », dont la pendularité entre villes, qui parfois implique une double résidence, fait partie. Ces longues mobilités pendulaires s'inscrivent, par ailleurs, dans une nouvelle catégorie de mobilités, dites réversibles ${ }^{1}$, qui s'opposent aux mobilités dites irréversibles que sont la migration ou le déménagement [10]. Les mobilités réversibles sont caractérisées par la longue distance, une faible temporalité et une faible mobilité sociale, et ont encore été peu étudiées, notamment d'un point de vue quantitatif.

La particularité de cet article est de se concentrer sur les déplacements domicile-travail de l'ensemble des actifs français habitant une aire urbaine de taille moyenne et ayant leur emploi en dehors de celle-ci. L'ensemble de ces liaisons ne sont pas de fait de longues distances. Elles peuvent être la conséquence d'un simple effet statistique lié à la construction des limites de l'aire urbaine (construction fonctionnelle basée sur les flux domicile-travail). Pour rappel, pour construire la couronne périurbaine d'une aire urbaine et ainsi définir les limites de celle-ci, l'Insee (Institut national de la statistique et des études économiques), prend en considération les communes dont au « moins $40 \%$ de la population résidente ayant un emploi travaille dans le pôle ou dans des communes attirées par celui-ci $»^{2}$.

\footnotetext{
${ }^{1}$ Site du Forum Vies Mobiles : http://fr.forumviesmobiles.org/ reperes/mobilites-reversibles-462 consulté le 23 juillet 2014 .

${ }^{2}$ Site de 1'Insee : http://www.insee.fr/fr/methodes/default.asp?page= definitions/aire-urbaine.htm consulté le 23 juillet 2014.
}

Toutefois l'analyse du recensement de la population de 2010 montre que les liaisons domicile-travail de courtes distances en marge des aires urbaines ne constituent pas la majorité des déplacements qui sortent d'une aire urbaine.

La seconde originalité de notre démarche est qu'elle porte sur des agglomérations moins étudiées par les recherches sur les mobilités domicile-travail : les villes moyennes. Dans cet article, nous définissons les villes moyennes par leur poids démographique : les aires urbaines ayant une population comprise entre 100000 et 500000 habitants. Les recherches sur les déplacements dans ces villes à l'échelle intra-urbaine montrent déjà une présence notable de l'automobile dans tous les espaces de l'aire urbaine $[11,12,13,14]$. De plus ces villes afffichent un taux d'actifs travaillant en dehors de leur aire urbaine de résidence plus important que pour les grandes agglomérations : $15 \%$ en moyenne contre moins de $5 \%$ en moyenne pour les dix plus grandes aires urbaines françaises.

L'objectif de cet article est d'apporter une vision complémentaire des approches centrées sur les déplacements intra-urbains, en se concentrant sur les trajets réalisés par les actifs qui sortent de leur aire urbaine de résidence pour se rendre sur leur lieu d'emploi (population que nous nommons : «actifs sortants »), à l'échelle des villes moyennes. Notre propos sera construit autour de deux questions : Quelles sont les pratiques de déplacement des actifs sortants, et notamment la place de la voiture ? Et quelles sont les possibilités de report vers des modes de transport alternatifs?

Notre but est d'apporter une connaissance détaillée de ces mobilités en regardant plus précisément : les professions et catégories socioprofessionnelles, les distances effectuées, les différents modes de transport utilisés, les localisations des actifs dans l'aire urbaine de résidence, la distribution spatiale des lieux d'emploi (ville centre, pôle urbain, espace périurbain ou rural) et les directions des flux (métropoles, villes moyennes, petites villes ou diffus). Ce travail permettra d'établir un premier état des lieux de ces déplacements et de questionner les distances parcourues et la place de l'automobile. Nous interrogerons ainsi les potentialités d'altermobilités des différentes liaisons en fonction des éléments précédemment décrits [15].

Pour commencer, nous préciserons le contexte et le cadre théorique dans lesquels se situe notre recherche, puis nous présenterons les éléments de méthode utilisés pour ce travail statistique. Les résultats de l'analyse globale de l'ensemble des villes moyennes seront ensuite présentés, suivis de leur analyse en termes de potentialité de report vers des modes alternatifs. 


\section{Les déplacements domicile-travail au départ des villes moyennes, angle mort des réflexions sur la mobilité durable}

\section{Des déplacements domicile-travail qui n'ont jamais été aussi longs}

Si aujourd'hui la part du motif domicile-travail baisse en termes de représentation des différents motifs de déplacements (21\%), ces trajets représentent encore $41 \%$ des distances parcourues par les Français d'après l'ENTD (Enquête nationale transport et déplacement) de 2008 [16]. Ils demeurent aussi « un levier d'action des politiques publiques, compte tenu de leur caractère contraint et stable dans le temps » et possèdent un rôle structurant dans les déplacements quotidiens des actifs mais aussi de l'ensemble de la population [17]. Une étude des déplacements des Franciliens, montre par exemple que lorsque les individus travaillent ils ont moins de chance de réaliser d'autres motifs de déplacement : « ces résultats traduisent la faible marge de manœuvre des actifs pour programmer des activités privées les jours où ils travaillent » [1].

Toujours d'après l'ENTD, en 2008 « le trajet moyen est de 14,7 kilomètres et demande 22,6 minutes. De 1982 à 2008, la distance et le temps du trajet moyen ont augmenté mais la progression de la durée est moins forte en raison de l'utilisation massive de la voiture qui est à l'origine des gains de temps de transport. Les trajets d'au moins $10 \mathrm{~km}$ représentent un trajet sur deux en 2008 contre un sur trois en 1982. La part des trajets longs a fortement augmenté, celle des 20 à 40 kilomètres par exemple a doublé, atteignant $20 \% »[18]$. Ces chiffres nous apprennent deux choses, d'une part le fort développement de l'usage de l'automobile et d'autre part l'allongement continu des distances domicile-travail permis en partie par celle-ci. Ce constat est d'autant plus fort pour les grandes agglomérations : " les distances domicile-travail, généralement supérieures à celles des autres déplacements locaux, continuent de s'allonger ainsi que la durée des trajets. En moyenne, aller et revenir du travail demande près de 50 minutes à un actif des grandes agglomérations contre 36 minutes ailleurs. La part des déplacements en voiture a gagné deux points, permettant d'aller plus loin dans le même temps. » [19]. Cet allongement participe de l'influence du travail sur la mobilité quotidienne des actifs : «par le jeu de la contrainte des distances parcourues, qui s'accentue à mesure que la longueur du trajet domicile-travail augmente, la distribution des activités autres devrait se concentrer le long du trajet domicile-travail et/ou à proximité $\mathrm{du}$ lieu de résidence. L'effet structurant serait donc d'autant plus sensible que la distance domicile-travail est grande » [20].
Les déplacements domicile-travail et les espaces périurbains au centre des enjeux de mobilité durable

Le lien entre les consommations énergétiques des déplacements quotidiens, la densité urbaine et la place de l'automobile est bien connu depuis les travaux de Newman et Kenwothy [21]. La faible densité incite l'usage de l'automobile et augmente les consommations énergétiques et les émissions de polluants. Ces conclusions ont été largement commentées et critiquées, mettant en avant le fait que la densité est un indicateur fruste et que l'usage de la voiture est aussi conditionné par la vitesse des réseaux, le degré de spécialisation sociale et technique des territoires, les contraintes de stationnement ou encore le revenu des ménages [22]. Malgré tout, l'objectif d'une baisse de l'usage de la voiture est relativement consensuel, et passe par quatre actions principales : baisser le nombre de trajet en voiture, réduire la longueur des trajets en automobile, encourager le report modal et favoriser le développement du système de transport en commun [23,24,25]. Les espaces périurbains sont particulièrement visés : en France, « un résident des centres urbains, denses et équipés en services et commerces de proximité, peut émettre jusqu'à trois fois moins de GES pour se déplacer qu'un résident des zones périurbaines de l'agglomération » [26].

\section{Les pendulaires travaillant en dehors de leur aire urbaine : plus émetteurs ?}

La distribution des distances des déplacements domiciletravail montre une proportion assez faible de longs déplacements en voiture qui pèsent par contre fortement sur la consommation énergétique et les émissions polluantes. On connaît toutefois encore mal la place des trajets domicile-travail des actifs sortant de leur aire urbaine de résidence sur cette distribution et donc leur importance dans le bilan environnemental (consommation énergétique et émissions de polluants). Le lieu d'emploi de ces actifs peut aussi bien se trouver dans un territoire rural que dans la couronne périurbaine, le pôle urbain ou la ville-centre d'une autre aire urbaine. Partant de leur commune de résidence, les points de départs des liaisons de ces actifs peuvent être de trois différents types d'espaces : la ville-centre, le pôle urbain et la couronne périurbaine. On renverse en partie la construction fonctionnelle des limites de l'aire urbaine, basée sur les flux centripètes des communes périurbaines, en prenant en compte l'ensemble des actifs qui la quittent. Les hypothèses qui nous poussent à porter notre regard sur ces relations sont d'une part qu'elles s'effectuent sur des distances plus longues qu'à l'intérieur des aires urbaines (en dehors des effets de bord : actif d'une commune rattachée à la limite de l'aire urbaine mais travaillant dans une 
commune voisine non rattachée à l'aire urbaine en question) et d'autre part qu'elles sont principalement effectuées en voiture. Ces liaisons apparaissent à la marge des études sur la durabilité des déplacements qui se concentrent le plus souvent sur les déplacements intra-urbains. Un récent travail sur les déplacements internes à la région Bourgogne montre que les déplacements interurbains sont plus problématiques en termes d'émission de $\mathrm{CO} 2$ car ils sont réalisés sur de grandes distances et principalement en automobile : « Le mode de transport principalement utilisé varie en fonction de la distance à parcourir, mais le véhicule motorisé individuel, en particulier la voiture, reste le mode de déplacement privilégié des Bourguignons. Il est utilisé par $80 \%$ des navetteurs. Les déplacements entre grandes villes [...] contribuent fortement aux rejets de dioxyde de carbone dans l'atmosphère en raison de la longueur des trajets. Ainsi, les $5,4 \%$ actifs occupés et étudiants bourguignons, qui effectuent chaque jour entre 50 et $200 \mathrm{~km}$, sont responsables de $28 \%$ du CO2 total émis » [17]. Ce dernier résultat est assez frappant et questionne largement les responsabilités de certains actifs dans le total des émissions du territoire. Les données de l'ENTD de 2008 viennent en appui de ces résultats et montrent la place importante de la voiture dans ces déplacements entre agglomérations : «En France, $18 \%$ des émissions totales des navettes sont le fait des 2000 liaisons entre agglomérations les plus émettrices (y compris au sein d'une même aire urbaine). Bien que s'effectuant entre zones denses, le recours à la voiture est massif, au détriment des transports collectifs. Les émissions correspondantes de $\mathrm{CO} 2$ par personne sont cinq fois plus élevées que pour les flux internes aux pôles urbains » [27]. Au-delà de leur poids dans ce total, ces déplacements interrogent également la durabilité sociale de ces déplacements en termes d'accessibilité à l'emploi (les actifs doivent quitter leur aire urbaine pour travailler) et de fragilité de ces liaisons (effectués en automobile dans un contexte de tension énergétique) [28]. La durabilité environnementale n'est pas le seul enjeu d'une meilleure connaissance des navettes de longue distance. D'autres problématiques se développent, concernant par exemple les motivations et les modes de vie des personnes concernés. Des travaux sur les pendulaires en transport en commun entre Paris-Rouen, Paris-Tours et entre villes suisses [29,30,9], attestent de logiques qui conduisent les actifs à faire de grandes distances pour travailler sans aboutir à une mobilité irréversible, notamment à cause de : " l'emploi et l'opinion du conjoint, l'histoire individuelle et le vécu, le caractère non-renégociable du logement et de la volonté de conserver une "qualité de vie" " [29]. Ces déplacements de grandes distances effectués en train n'ont pas de représentativité nationale mais apportent des informations sur la raison qui poussent ces actifs à quitter leur aire urbaine de résidence, l'emploi, et sur celles qui les poussent à faire ces trajets quotidiennement.

\section{Les villes moyennes comme échelon pertinent d'étude de ces liaisons}

Dans cet article, nous entendons par villes moyennes les aires urbaines de la France métropolitaine dont la population est comprise entre 100000 et 500000 habitants. Elles sont au nombre de 68 en 2008. Ce seuil démographique n'est pas utilisé par toutes les études. Pour définir ces villes, aujourd'hui, " il existe presque autant de seuils que de chercheurs ou d'organismes en charge de collecter et de traiter des données sur ces villes » [31]. Nous ne ferons donc pas ici un inventaire de l'ensemble des seuils de population, parfois faites à l'échelle de la ville-centre, d'autres fois à celle de l'aire urbaine, ou encore d'autres au croisement des deux. Beaucoup de seuils existent et certains travaux possèdent le même que celui de notre étude $[12,13,32]$. Notons également que la problématique des actifs sortants n'est pas une question spécifique aux villes moyennes. Toutes les aires urbaines ont des actifs qui travaillent en dehors de l'aire urbaine de résidence. Nous avons choisi de nous concentrer sur ces villes pour plusieurs raisons. Tout d'abord, la place déjà forte de l'automobile dans ces villes à l'échelle des déplacements intra-urbains en raison de réseaux de transports en communs moins développés et de réseaux routiers moins congestionnés que dans les grandes villes. Un travail récent sur la mobilité dans les aires urbaines de 100000 à 500000 habitants montre que « la voiture, certes globalement plus discrète et en baisse dans les zones centrales, demeure néanmoins, en ville moyenne, infiniment plus répandue dans tous les tissus urbains que dans le cas des plus grandes villes. Son usage y est moins discriminé spatialement parce que l'espace est moins différencié (notamment les densités) » [13]. La voiture est partout présente et utilisé par tous. Pour les déplacements au lieu de travail, celle-ci est utilisée aussi bien dans le pôle urbain (79\%), que dans l'espace périurbain (78\%), alors que les transports en communs ne sont utilisés que par $8 \%$ des actifs dans le pôle urbain et $2 \%$ dans la couronne périurbaine. Ensuite, les villes moyennes diffusent une part d'actifs plus importante que les grandes aires urbaines. Associées à l'attrait lié au foncier, les villes moyennes manquent d'emplois très qualifiés ou d'emploi que l'on ne trouve que dans les grandes aires urbaines. Ces facteurs peuvent encourager certains actifs vers des déplacements entre villes : «En ville moyenne, la rareté des emplois qualifiés conduit une part des actifs à des pratiques de déplacement interrégionales, alors que la forte présence et concentration spatiale de ces emplois à Paris par exemple les rendent beaucoup plus accessibles aux populations locales » [13]. Enfin, elles regroupent un nombre assez conséquent d'actifs pour imaginer la mise en place d'alternatives crédibles à « l'autosolisme ». Les solutions envisageables peuvent être d'ordre divers, avec des 
caractéristiques de fréquence et de capacité différentes mais toutes nécessitent un minimum de voyageurs.

\section{Le recensement de la population comme source des déplacements domicile-travail}

Nos résultats reposent sur l'étude statistique des données de l'Insee à partir du fichier « Mobilités Professionnelles » de 2010. Celui-ci permet de saisir les déplacements domiciletravail des actifs français à partir de leur commune de résidence et d'emploi. Comme déjà évoqué, l'échelle prise en compte est celle des aires urbaines. Nous reprendrons le découpage comme l'Insee le définit : « une aire urbaine est un ensemble de communes, d'un seul tenant et sans enclave, constitué par un pôle urbain (unité urbaine) de plus de 1000 emplois, et par des communes rurales ou unités urbaines (couronne périurbaine) dont au moins $40 \%$ de la population résidente ayant un emploi travaille dans le pôle ou dans des communes attirées par celui-ci $»^{3}$. Pour prendre en compte cette échelle de travail nous utilisons le fichier du zonage en aire urbaine de l'Insee de $2010^{4}$ qui nous permet de disposer des informations sur ce zonage pour chaque commune, et de savoir par exemple, si elles appartiennent à une aire urbaine ou non, et si oui, à laquelle.

Toutefois ce traitement est apparu incomplet pour notre étude. Nous avons alors construit deux nouveaux zonages : un hiérarchique et le second typologique.

Le regroupement hiérarchique est une simplification du découpage géographique de l'Insee intitulé « Tranche d'aire urbaine $2010 »$ qui indique la fourchette de la taille de l'aire urbaine à laquelle la commune appartient. Ce découpage qui est constitué de 11 tranches sera réduit à 5 niveaux :

- les communes appartenant à l'aire urbaine de Paris ;

- les communes appartenant à une grande aire urbaine : aire urbaine dont la population regroupe plus de 500000 habitants, hors Paris ;

- les communes appartenant à une aire urbaine de ville moyenne : aire urbaine dont la population regroupe entre 100000 et 500000 habitants ;

- les communes appartenant à une aire urbaine petite : aire urbaine dont la population est inférieure à 100000 habitants ;

- les communes multipolarisées ou hors aire urbaine.

Le second zonage reprend le découpage de l'Insee intitulé « Catégorie de la commune dans le zonage en aires

\footnotetext{
${ }^{3}$ Site de 1'Insee : http://www.insee.fr/fr/methodes/default.asp?page $=$ definitions/aire-urbaine.htm consulté le 23 juillet 2014 .

${ }^{4}$ Site de 1'Insee : http://www.insee.fr/fr/methodes/default.asp?page= zonages/aires_urbaines.htm consulté le 23 juillet 2014.
}

urbaines $2010 »$ qui permet de savoir si la commune est périurbaine, appartenant à un pôle urbain, multipolarisée ou hors influence d'un pôle urbain. L'Insee construit 9 modalités de communes, nous en choisissons 5 :

- les communes « ville-centre » qui correspondent à la commune centrale de l'aire urbaine ${ }^{5}$;

- les communes appartenant au pôle urbain d'une aire urbaine ;

- les communes appartenant à l'espace périurbain d'une aire urbaine ;

- les communes multipolarisées.

- Les communes isolées hors influence des pôles.

Les données du recensement de 2006, 2007 et 2008 ont été découpées suivant les mêmes catégories, en fonction du zonage en aire urbaine de 2010. Il en est de même pour les chiffres du RGP (Recensement général de la population) de 1999 qui a été découpé suivant les mêmes limites de 2010 afin de faciliter les comparaisons entre ces dates. Notons également que l'ensemble des distances dans cet article sont calculées à vol d'oiseau et que les trajets infracommunaux sont considérés comme nuls du fait de l'absence d'information. De plus, nous prenons en compte l'intégralité des flux domicile-domicile travail au départ des aires urbaines à l'exception des flux en direction : de l'étranger, des DOM-TOM et de la Corse. L'ensemble des villes moyennes étudiées rassemble 68 aires urbaines de France métropolitaine (Fig. 1). Les figures et tableaux présentés dans cet article sont tous issus des données fournies par l'Insee.

\section{Les mobilités domicile-travail au départ des villes moyennes}

Un bref retour sur les recensements précédents atteste que les trajets domicile-travail qui sortent d'une aire urbaine ne sont pas un phénomène nouveau, et qu'il tend à s'intensifier. Pour rappel, les chiffres présentés sont tous calculés suivant le zonage en aire urbaine de l'Insee de 2010. Nous restons prudents dans la comparaison avec les données de 1999 mais les données confirment néanmoins une tendance : depuis 1999 la part des actifs sortants des villes moyennes par rapport à l'ensemble de la population active résidente est en augmentation. Les chiffres du tableau suivant (Tableau 1) montrent que l'on passe de 13,2 \% d'actifs sortants en 1999

\footnotetext{
5 Pour les aires urbaines qui sont composées du nom de deux communes, comme Douai-Lens ou Marseille-Aix-en-Provence, les deux communes sont enregistrées comme « ville-centre».
} 


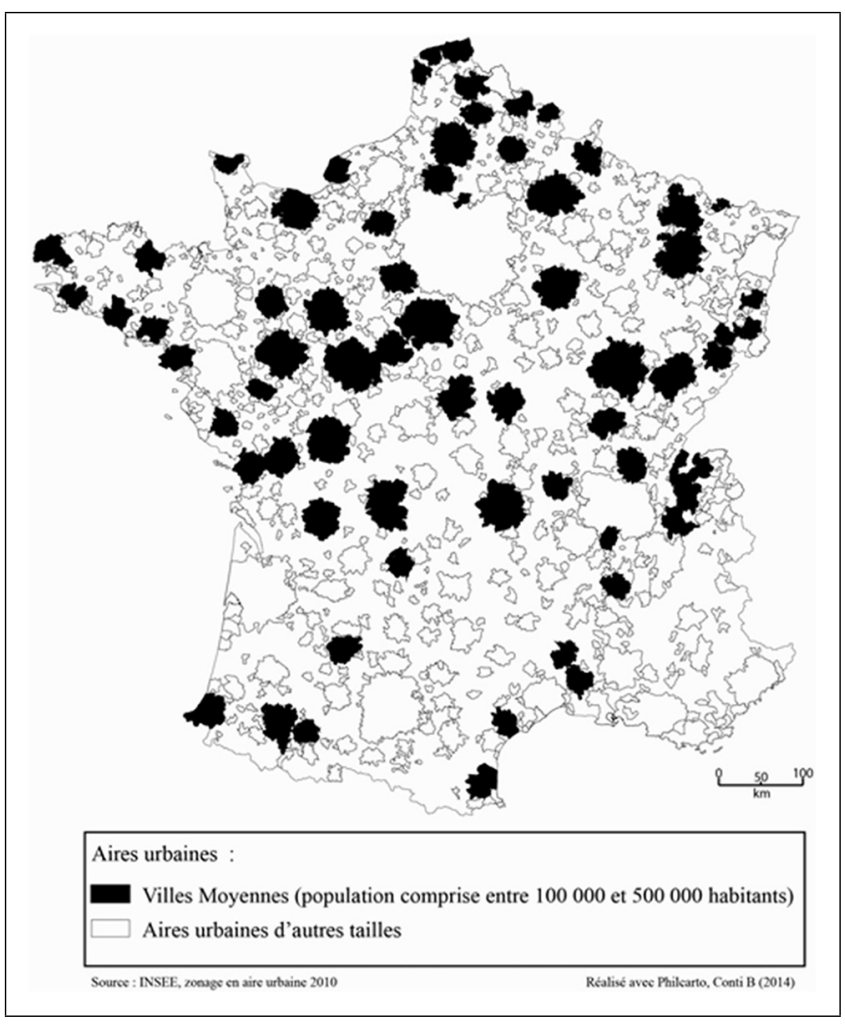

Fig. 1 Les 68 aires urbaines des villes moyennes étudiées

à $15,2 \%$ en 2010 , soit une augmentation en valeur absolue de presque 200000 actifs en un peu plus de 10 ans.

\section{Peu d'actifs responsables de beaucoup de distances}

En 2010, les actifs des 68 aires urbaines des villes moyennes représentent un peu plus de 5700000 personnes et ont une distance moyenne domicile-travail à vol d'oiseau de 14,1 km (Tableau 2). Parmi eux, les actifs sortants ne comptent que pour $15 \%$, soit un peu plus de 873000 actifs. Un premier résultat important concerne la comparaison de ce taux d'actifs sortants ( $15 \%$ ) avec la part qu'ils représentent par rapport à l'ensemble des kilomètres parcourus par tous les actifs : $67 \%$. Cela signifie que $15 \%$ des actifs effectuent un plus des deux tiers des distances parcourues par tous les actifs résidents, et cela en lien avec des distances moyennes beaucoup plus élevées $(62 \mathrm{~km})$ que pour les déplacements à l'intérieur de l'aire urbaine $(5,4 \mathrm{~km})$. C'est un premier résultat intéressant qui nous porte à croire qu'il faut s'intéresser davantage à ce type de flux : ces actifs sortants sont peu nombreux, effectuent en moyenne de grandes distances et sont responsables de beaucoup de kilomètres.

La dispersion de la distribution des distances des actifs sortants est bien plus grande que pour les actifs navettant dans les aires urbaines. Le détail de cette dispersion révèle que la médiane des actifs sortants est de $28,2 \mathrm{~km}$, la moyenne de $62,1 \mathrm{~km}$ et que $90 \%$ des actifs font moins de $138 \mathrm{~km}$. Ces déplacements ne sont donc pas de simple effet de bord, c'est-à-dire qu'ils ne sont pas le fruit d'actif résidant aux limites des aires urbaines et effectuant de courtes distances en dehors de celles-ci. Ces trajets existent sans doute, mais ne représentent pas la majorité des déplacements que nous observons dans ce travail. Une analyse plus précise du taux d'actifs sortant nous apporte d'autres informations sur les différences entre les aires urbaines. Ce taux peut en effet varier du simple au double : parfois il dépasse $20 \%$ (Cherbourg, Creil, Colmar) alors que dans d'autres villes il est inférieur à $10 \%$ (Bayonne, Perpignan, Orléans). L'explication de ces écarts relève tant de caractéristiques individuelles que territoriales qui ne sont pas abordées ici. Notons simplement que plus il y a d'actifs qui quittent leur aire urbaine de résidence pour travailler, plus ils représentent une part élevée dans le total des distances effectuées par l'ensemble des actifs de l'aire urbaine.

\section{Une mobilité largement tournée vers la voiture, sauf vers Paris}

Le tableau suivant (Tableau 3) confirme que la voiture est prépondérante dans les déplacements des actifs sortants. En moyenne, $85 \%$ des actifs utilisent la voiture pour se rendre sur leur lieu d'emploi alors qu'ils ne sont que $12 \%$ à utiliser les transports en commun. On notera qu'en termes de part des distances dans le total parcouru, ce pourcentage évolue fortement puisqu'il passe respectivement à $70 \%$ pour la voiture et $23 \%$ pour les transports en commun. La voiture est davantage utilisée pour les trajets réalisés à proximité (distance moyenne de $51 \mathrm{~km}$ ) alors que les transports en commun le sont plus pour les plus longues distances (distance moyenne de $123 \mathrm{~km}$ ).

\begin{tabular}{|c|c|c|c|c|c|}
\hline Année & 1999 & 2006 & 2007 & 2008 & 2010 \\
\hline Nombre d'actifs sortants & 678479 & 819499 & 839504 & 850750 & 873807 \\
\hline Part d'actifs sortants & $13,2 \%$ & $14,5 \%$ & $14,7 \%$ & $14,9 \%$ & $15,2 \%$ \\
\hline
\end{tabular}


Tableau 2 Comparaison des caractéristiques générales des distances entre actifs sortant et actifs résidant et travaillant dans l'aire urbaine

\begin{tabular}{lcccc}
\hline & Distance moyenne & Nombre d'actifs & Part des actifs & Part des distances \\
\hline Sortant $\mathrm{AU}^{*}$ & $62,1 \mathrm{~km}$ & 873808 & $15 \%$ & $67 \%$ \\
Intra AU* & $5,4 \mathrm{~km}$ & 4841092 & $85 \%$ & $33 \%$ \\
Ensemble & $14,1 \mathrm{~km}$ & 5714900 & $100 \%$ & $100 \%$ \\
\hline AU*: aire urbaine. & & & \\
\hline
\end{tabular}

Tableau 3 Mode de transport des actifs sortants pour les navettes domicile-travail

\begin{tabular}{lccrr}
\hline & Distance moyenne & Nombre d'actifs & Part des actifs & Part des distances \\
\hline Voiture & $51,3 \mathrm{~km}$ & 744036 & $85 \%$ & $70 \%$ \\
TC & $123,0 \mathrm{~km}$ & 103062 & $12 \%$ & $23 \%$ \\
Autres modes & & 26710 & $4 \%$ & $7 \%$ \\
\hline
\end{tabular}

Des distinctions territoriales existent toutefois entre les aires urbaines, avec des villes où la voiture est bien plus utilisée que les transports en commun. La relation à Paris et la distance parcourue apparaissent comme des variables ayant un rôle important sur le choix du mode de transport des actifs. Plus une aire urbaine est en relation avec Paris, plus les actifs utilisent les transports en commun, et, un actif qui effectue de grandes distances a moins de chance d'utiliser sa voiture pour se rendre sur son lieu de travail.

Les résultats sur les professions et catégories socioprofessionnelles sont plutôt semblables à ce que l'on peut observer dans les études sur les déplacements quotidiens des PCS (Professions et catégories socioprofessionnelles) : les actifs sortants cadres effectuent les distances les plus longues, $84 \mathrm{~km}$ en moyenne, et de manière mécanique leurs trajets comptent plus dans la part totale des distances (27\%) par rapport à leur représentation par rapport à tous les actifs $(20 \%)$. Ces résultats viennent en écho de résultats suivants qui montrent des liaisons différentes avec des aires urbaines plus ou moins importantes en fonction des PCS.

\section{Des liaisons au départ d'une variété de lieu vers des espaces denses}

Les données du tableau 4 font apparaître le poids des distances des différentes navettes par rapport à l'ensemble des liaisons possibles. Le total du tableau est égal à $100 \%$. Celui-ci nous apprend une chose intéressante : la grande majorité des distances s'effectuent vers des zones ayant une certaine densité. $81 \%$ des trajets domicile-travail des actifs sortants s'effectuent soit vers des communes ville-centre (50\%), soit vers des communes d'un pôle urbain (31\%). Ces liaisons sont effectuées à $29 \%$ depuis une commune ville-centre, à $26 \%$ depuis le pôle urbain et également à $26 \%$ depuis le pôle urbain.

Si l'on regarde les modes de transports utilisés en fonction du lieu d'emploi et du lieu de résidence, on peut faire deux constats : plus les actifs habitent une ville-centre, plus ils ont tendance à utiliser les transports en commun ; plus les actifs vont travailler dans une commune ville-centre, plus ils ont de chance de s'y rendre en transport en commun. Pour les liaisons de « commune ville-centre vers commune ville-centre », la voiture est utilisée par $70 \%$ des actifs, alors que, par exemple, pour les liaisons au départ de communes périurbaines (vers les villes-centres ou les pôles urbains) elle est utilisée par $90 \%$ des actifs. Même si dans tous les cas de figure la voiture reste le mode de transport majoritaire, ces résultats laissent à penser que des marges de manœuvre sont possibles pour développer les altermobilités. Le fait que les actifs se dirigent principalement vers des zones relativement denses et centrales porte à croire que d'autres formes de mobilité que l'autosolisme sont envisageables.

\section{Paris et la hiérarchie urbaine jouent des rôles différenciés}

La grande majorité des actifs vont travailler dans une aire urbaine (77\%) et un quart ont un emploi en dehors (dans une commune multipolarisées ou hors influence d'un pôle urbain) (Tableau 5). Si l'on s'attarde sur la distribution des 


\begin{tabular}{|c|c|c|c|c|}
\hline & & \multicolumn{3}{|c|}{ Commune de résidence } \\
\hline & & Ville-centre & Pôle urbain & Périurbain \\
\hline \multirow[t]{5}{*}{ Commune de travail } & Ville-centre & $19 \%$ & $15 \%$ & $16 \%$ \\
\hline & Pôle urbain & $10 \%$ & $11 \%$ & $10 \%$ \\
\hline & Périurbain & $2 \%$ & $2 \%$ & $3 \%$ \\
\hline & Multipolarisé & $2 \%$ & $2 \%$ & $3 \%$ \\
\hline & Hors influence & $2 \%$ & $1 \%$ & $2 \%$ \\
\hline
\end{tabular}

Tableau 5 Des caractéristiques différentes en fonction de l'aire urbaine de travail

\begin{tabular}{lcll}
\hline & $\begin{array}{l}\text { Distance } \\
\text { moyenne }\end{array}$ & $\begin{array}{l}\text { Part des } \\
\text { actifs }\end{array}$ & $\begin{array}{l}\text { Part des } \\
\text { distances }\end{array}$ \\
\hline Paris & $167 \mathrm{~km}$ & $10 \%$ & $28 \%$ \\
Grande AU & $80 \mathrm{~km}$ & $16 \%$ & $20 \%$ \\
Moyenne AU & $70 \mathrm{~km}$ & $16 \%$ & $18 \%$ \\
Petite AU & $40 \mathrm{~km}$ & $35 \%$ & $22 \%$ \\
Hors AU & $32 \mathrm{~km}$ & $23 \%$ & $12 \%$ \\
\hline
\end{tabular}

actifs, $35 \%$ vont dans une aire urbaine de moins de 100000 habitants, $16 \%$ dans une autre ville moyenne, $16 \%$ encore vont vers des aires urbaines avec une population supérieure à 500000 habitants et Paris attire $10 \%$ des actifs sortant des villes moyennes.

Si l'on raisonne en termes de distance, ce sont les villes de niveau équivalent ou supérieur qui génèrent le plus de kilomètres. L'ensemble des aires urbaines de travail ayant une population supérieure à 100000 habitants attirent $42 \%$ des actifs qui réalisent $66 \%$ de l'ensemble des distances parcourues. Les flux sont davantage dirigés vers des espaces plutôt centraux, comme nous avant vu précédemment, et vers des aires urbaines de niveau équivalent ou supérieur.

D'autres résultats sur les modes de transport utilisés nous apprennent que la part des transports en commun est largement portée par les déplacements vers la capitale. Les actifs se rendant dans l'aire urbaine parisienne sont $42 \%$ à utiliser les transports en commun alors que pour se rendre dans les autres niveaux de la hiérarchie urbaine, la voiture est utilisée par au moins $80 \%$ des actifs. Plus les actifs se dirigent vers des villes qui sont hautes dans la hiérarchie urbaine, plus ils ont tendance à parcourir de grandes distances et à utiliser toujours massivement la voiture (sauf vers l'aire urbaine de Paris).

L'analyse par PCS montre qu'on ne constate pas d'effet de la hiérarchie urbaine pour les professions intermédiaires et les employés, qui vont proportionnellement autant travailler dans les aires urbaines de petites tailles que vers les grandes et Paris. En revanche, pour les cadres et les ouvriers la différence est manifeste : les premiers sont surreprésentés dans les actifs allant vers Paris alors que les seconds sont surreprésentés dans les actifs travaillant hors des aires urbaines. Ces chiffres sont à mettre en regard de la structure des emplois occupés, avec une surreprésentation des emplois cadres dans la capitale et les grandes villes et plus d'emplois de type ouvriers dans les espaces moins denses du territoire.

Si l'on rapporte ces résultats à une analyse plus précise, les taux d'actifs sortant et donc les distances parcourues par ces actifs, ne sont pas forcément en lien avec le niveau de la hiérarchie urbaine. C'est-à-dire que les villes qui ont un taux d'actifs sortants important n'émettent pas forcément vers Paris, et dans les villes où ce taux est plus faible, les actifs ne vont pas forcément vers des espaces diffus. Pour citer quelques exemples, certaines villes moyennes envoient beaucoup d'actifs vers des grandes villes (Valenciennes), des villes de niveau équivalent (Thionville), des petites villes (Quimper) ou vers du diffus (Cherbourg).

\section{Quelles perspectives d'alternative à l'autosolisme pour ces déplacements ?}

« La maîtrise des déplacements automobiles est un enjeu fort des politiques publiques, quels que soient les niveaux de gouvernance. Son importance est notamment liée aux conséquences néfastes des déplacements automobiles sur l'environnement »[15]. Ces propos reprennent tout un ensemble d'études mettant en évidence la responsabilité 
de la voiture dans le bilan environnemental de la mobilité quotidienne. L'enjeu de maitrise de ces déplacements en automobile passe par la promotion d'un usage différent de la voiture ou par un transfert modal, « l'altermobilité ne signifie donc pas la fin de la voiture, mais plutôt un affaiblissement du « réflexe » automobile. » [15]. Comment maîtriser les déplacements automobiles des actifs sortants en affaiblissant la part modale de celle-ci ?

La question du report modal est complexe et fait entrer en jeu de nombreux facteurs que nous n'avons pas intégrés dans cet article comme par exemple les variables de temps, de coût, de représentations et de confort des déplacements. Elles sont pourtant déterminantes dans le choix que font ces actifs pour préférer un mode à un autre et peut-être encore plus pour le cas des déplacements longs des actifs sortants. Pour eux les distances sont plus longues que pour les déplacements intra-aire urbaine et la flexibilité temporelle semble être une variable clé [33]. La voiture peut apparaitre alors comme le mode le plus performant et le plus flexible (capacité de faire du porte à porte, liberté horaire, possibilité de chaîner plusieurs motifs). Les perspectives de report modal envisagées ici ne sont faites qu'à partir des résultats établis sur les distances et types de liaisons des actifs sortants tout en gardant à l'esprit que d'autres facteurs interviennent sur le choix modal. A priori une seule et unique politique de transport ne paraît pas à même de proposer de solution pour l'ensemble des différentes liaisons possibles. Ainsi, comment les déplacements des actifs sortants décrits dans cet article rencontre les politiques de transport actuellement en place en France ?

Les TET (Trains d'équilibre du territoire) apparaissent comme une solution envisageable pour relier les villescentre des villes moyennes vers d'autres espaces centraux compte tenu de leur pertinence en termes de trafic attendu. Aujourd'hui les TET « représentent 40 lignes exploitées sous 4 marques différentes (Corail, Intercités, Téoz et Lunéa), soit 340 trains par jour, desservant 367 villes et utilisés par environ 100000 passagers quotidiennement. Il s'agit de trains de moyenne et grande distances qui assurent des missions nationales ${ }^{6}$. À l'échelle régionale, le TER (Transport express régional) peut permettre d'assurer des liaisons entre communes de pôles urbains distincts. En parallèle, le bus interurbain apparaît comme une solution envisageable pour le même type de liaison (d'espaces denses vers un autre espace dense) que les TER pour les villes avec moins de voyageurs. Mais, les bus interurbain, souvent

\footnotetext{
${ }^{6}$ Site internet du MEDDE (Ministère de l'Écologie du Développement Durable et de l'Énergie) : http://www.developpementdurable.gouv.fr/La-convention-TET-un-engagement.html consulté le 23 juillet 2014.
}

utilisés dans d'autres pays, sont en France majoritairement des bus à vocation scolaire sous la responsabilité des départements : « en 2009, l'enquête Transports Collectifs Départementaux (enquête TCD) du Certu, a estimé que $79 \%$ de la fréquentation des lignes régulières de voyageurs sur les transports départementaux est réalisée par des scolaires » [34]. De plus, ils ont des compétences réduites en milieu urbain, avec l'interdiction de prendre en charge des voyageurs dans le périmètre des AOTU (Autorité organisatrice des transports urbains) [35].

Pour les liaisons au départ du périurbain vers les pôles urbains, les TER prennent place dans la desserte de ces zones périphériques [36]. Toutefois ces liaisons ne sont envisagées que depuis des espaces périurbains vers la ville-centre de l'aire urbaine dont ils dépendent et non vers le pôle urbain d'une autre aire urbaine [37]. Pour les cas où le réseau régional permet un accès aux différentes aires urbaines, des navettes de rabattement vers le réseau ferré ou routier interurbains peuvent être possibles. Ce type d'expérimentation a par exemple été retenu par le schéma de mobilité du département du Nord, avec des horaires « calquées sur les horaires des TER » [38]. Toutefois ces liaisons doivent être envisagées pour le cas où le réseau régional assure bien une jonction entre différentes aires urbaines.

Des actifs sortants se dirigent aussi vers des espaces périurbains tout en habitant le centre, le pôle urbain ou l'espace périurbain. Pour ces derniers, les politiques de transport en faveur du covoiturage semblent les mieux adaptées. Le covoiturage permet de regrouper les actifs afin de diminuer les émissions polluantes tout en mutualisant les coûts de déplacement et apparaît comme une vraie solution d'altermobilité pour affaiblir l'autosolisme [39]. Il est souvent mis en avant comme la solution de transport à partir d'espaces peu denses, mais en regard des liaisons observées ici, on peut l'envisager pour des trajets en direction de communes périurbaines.

Plusieurs niveaux et solutions de transport sont donc a priori possibles, et ce, en fonction des différents types de liaisons. Néanmoins, notre propos est ici globalisant et n'est pas forcément envisageable pour toutes les liaisons au départ de toutes les villes moyennes. Le contexte local comme le type de relation (par exemple avec des métropoles ou des villes plus petites) sur de plus ou moins grandes distances, avec un plus ou moins grand nombre d'actifs jouent sur la portée de notre analyse. Toutefois, il apparaît possible d'étoffer le champs des possibles offert aux actifs sortants par une meilleure compréhension et prise en compte de leur pratique de mobilité, principalement focalisée aujourd'hui sur les déplacements intra-urbains. 


\section{Conclusion}

Cinq résultats importants sont à retenir de l'analyse des déplacements des actifs sortant des villes moyennes : ils sont peu nombreux ( $15 \%$ ) mais font beaucoup de distances, donc nous ne sommes pas dans le cas de simples effets de bord ; ils utilisent majoritairement l'automobile pour leur navette quotidienne ( $85 \%$ en moyenne) ; ils résident dans tous les espaces des aires urbaines [40] mais vont principalement travailler dans des espaces denses (la ville-centre d'une aire urbaine ou son pôle urbain), où se concentrent les emplois ; les modes de transports et les distances sont légèrement différents selon les liaisons : les actifs utilisent davantage les transports en commun pour se rendre dans une ville-centre d'une grande aire urbaine par exemple ; enfin l'ensemble des PCS sont concernés par ces liaisons, pas simplement les cadres supérieurs.

Ces résultats attestent de modes de vie spécifiques de ces actifs, qui effectuent des distances plus importantes que les autres et questionnent de nouveau la conjoncture de Zahavi. Les travaux de Joly sur les Budgets temps de transports (BTT) mettent en évidence cette place forte de l'automobile qui ne conduit pas une stabilisation du BTT mais au contraire, à une augmentation des distances mais aussi des temps passés en transport [41]. Ces conclusions renforcent notre analyse pour le cas des actifs sortants et de leur proportion à faire des distances plus grandes que les autres (actifs résidant et travaillant dans les aires urbaines), et font écho aux résultats des travaux du programme de recherche JobMob sur les grands navetteurs ( $2 \mathrm{~h}$ Aller-retour minimum) [42].

Les déplacements des actifs sortants soulèvent également un véritable enjeu de durabilité (consommations énergétiques et émission de polluants) qui est aujourd'hui largement confiné aux déplacements intra-urbains. Ces actifs, peu nombreux, effectuent pourtant un nombre conséquent de kilomètres en automobile par rapport à l'ensemble des actifs. Ces résultats sont toutefois à mettre perspective de travaux sur la mobilité loisirs qui peut jouer comme mécanisme de compensation des émissions liées aux mobilités domicile-travail. Les émissions liées aux loisirs en lien avec la localisation résidentielle et le rapport au cadre de vie peuvent influer sur le bilan total d'émissions de polluants de ces actifs [43].

Enfin, cette étude ne nous permet pas d'étoffer la connaissance sur les motivations des actifs à effectuer ce type de liaisons. Des analyses qualitatives auprès d'eux apporteraient une richesse à nos travaux et à leur mise en perspective pour saisir les raisons qui les poussent à faire ces déplacements. En regard de nos résultats exploratoires sur ce sujet, deux groupes de variables semblent identifiables. Le premier a trait davantage au contexte local, aux caractéristiques territoriales (par exemple la position géographique sur le territoire français), à la présence d'une infrastructure de transport et aux caractéristiques des déplacements [31,35]. Le second est en lien avec des enjeux davantage individuels, liés notamment au parcours de vie et aux caractéristiques des actifs [44]. Dans toutes les études sur les grands navetteurs, l'emploi semble être la source principale qui conduit les actifs à effectuer ce type de déplacement alors que la situation familiale [45], la bi-activité du ménage [7], l'ancrage social $[9,46]$, « le caractère non-négociable du logement et la volonté de conserver une "qualité de vie" "[29], semblent être les raisons qui les poussent à ne pas les conduire vers un déménagement à proximité du lieu d'emploi.

L'ensemble des résultats présentés sont à mettre en perspective des choix méthodologiques. Comme évoqué précédemment, nous avons choisi de prendre en compte l'ensemble des flux au départ des villes moyennes, sans distinction. Or quelques trajets recensés dans les données Insee portent à croire que certaines liaisons sont difficilement réalisables avec aller-retour dans une journée de travail. Dans le cadre de cette phase exploratoire de l'étude de ces mobilités, l'ensemble des liaisons ont été conservées. Il serait intéressant par la suite de discriminer ces liaisons en fonction de distance et temps de transport. Le manque d'information des données de l'Insee sur les fréquences de ces déplacements (quotidiennes et hebdomadaires), tout comme les possibilités de télétravail [32] ou l'absence de données sur la bi-résidentialité [9], sont des facteurs qui peuvent jouer sur la comparaison entre les actifs sortants et l'ensemble des actifs et justifient le besoin d'études complémentaires. Enfin, les villes moyennes sont présentées de manière assez homogène dans nos résultats alors que l'influence spatiale locale de ces mobilités joue sans doute un grand rôle : en témoignent les taux d'actifs sortant différents entre chaque aire urbaine, la direction de ces flux et le type de relation avec le niveau hiérarchique des aires urbaines. L'analyse des différences entre villes constitue donc une piste de recherche intéressante.

\section{Références}

1. Aguiléra A, Massot M-H, Proulhac L (2010) « Travailler et se déplacer au quotidien dans une métropole. Contraintes, ressources et arbitrages des actifs franciliens ». Sociétés Contemporaines 80.

2. Donzelot J (2004) La ville à trois vitesses. Esprit, Mars

3. Dupuy G (2006) La dépendance à l'égard de l'automobile. PREDIT, La Documentation française.

4. Van der Laan L (1998) Changing Urban Systems: An Empirical Analysis at Two Spatial Levels. Regional Studies 32(3) : 235-247

5. Bertaud A, Lefevre B, Yuen B (2009) GHG Emissions, Urban Mobility and Efficiency of Urban Morphology: A Hypothesis. Urban Research Symposium, June 28-30, Marseille, France 
6. Aguiléra A (2010) Urban Form and commuting: A critical review of literature, 12th WCTR, July 11-15, Lisbon, Portugal

7. Laneelle X (2006) « Navette domicile-travail à grande vitesse : situation d'exception, arrangement traditionnel ». Cahiers $d u$ Genre, 2006/2:41, pp159-180

8. Kaufmann V (2008) Les paradoxes de la mobilité, bouger, s'enraciner. Presses polytechniques et universitaires romandes, Lausanne.

9. Kaufmann V (2010) Pendulaires ou bi-résidentiels en Suisse : un choix de vie ?. In Authier J-Y, Bonvalet C, Lévy J-P Elire Domicile, Presses Universitaires de Lyon, Lyon, pp 169-184.

10. Vincent-Geslin S, Kaufmann V (2012) Mobilités sans racine. Plus loin, plus vite... Plus mobiles? Éditions Descartes \& Cie.

11. De Roo P (2007) Les villes moyennes françaises. Enjeux et perspectives. Collection Travaux, DIACT.

12. Nadou F (2010) La notion de «villes intermédiaires », une approche différenciée du rôle des villes moyennes : entre structuration territoriale et spécificités socio-économiques. Colloque Villes petites et moyennes, un regard renouvelé, Tours, France

13. Massot M-H, Wenglenski S (2011) Prospective de la mobilité dans les villes moyennes. Futuribles Internationales, Rapport Final de l'étude

14. Floch J-M, Morel B (2011) Panorama des villes moyennes. Insee, Direction de la Diffusion et de l'Action régionale, Document de travail

15. Vincent-Geslin S (2010) Altermobilités, mode d'emploi. Déterminants et usages de mobilités alternatives au tout voiture. Certu, Lyon.

16. Le Jeannic T (2010) La mobilité des Français Panorama issu de l'enquête nationale transports et déplacements 2008. La Revue du $C G D D$, Décembre.

17. Brion D, Leger M (2012) Pour aller travailler ou étudier, les Bourguignons émettent 474000 tonnes de $\mathrm{CO} 2$ par an. Dimensions Insee Bourgogne 175.

18. François D (2010) Se rendre au travail : distances et temps de transport s'allongent. La Revue du CGDD, Décembre : 83-98

19. Hubert J-P (2009) Dans les grandes agglomérations, la mobilité quotidienne des habitants diminue, et elle augmente ailleurs. Insee Première 1252.

20. Boulahbal M (2001) Effet Polarisant du lieu de travail sur le territoire de la vie quotidienne des actifs. Recherche Transports Sécurité, Octobre-Décembre : 43-63.

21. Newman P-W- G, Kenworthy J-R (1989) Gasoline consumption and cities. A comparison of U.S. cities with a global survey. Journal of the American Planning Association 55(1) : 24-37.

22. Wiel M (2010) Etalement urbain et mobilité. PREDIT, Documentation Française.

23. Cervero R, Kockelman K (1997) Travel demand and the 3Ds: density, diversity, and design. Transportation Research D 2(3) : 199-219.

24. Banister D (2008), The sustainable mobility paradigm. Transport Policy $15: 73-80$.

25. Gori S, Nigro M, Petrelli M (2012) The impact of land use characteristics for sustainable mobility: the case study of Rome. European Transport Research Review 4 : 153-166.

26. Caubel D (2007) Réduire les disparités d'accès à la ville ? Une réponse concrète, mais imparfaite, par les transports collectifs. Les Cahiers Scientifiques du Transport 51 : 9-36.

27. Besson V, Durieux S, Levy D, Le Jeannic T (2012) Estimation des émissions de $\mathrm{CO} 2$ des navettes quotidiennes. Document de travail
28. Desjardins X (2011) Pour l'atténuation du changement climatique, quelle est la contribution possible de l'aménagement du territoire? Cybergeo : European Journal of Geography, Aménagement, Urbanisme, article 523.

29. Meissonnier J (2001) Provincilien : les voyageurs du quotidien, entre capitale et province, collection « Logique Sociale », L'Harmattan, Paris.

30. Beauvais J-M, Fouquet J-P, Assegond C (2007) Recherche sur le développement de la grande vitesse et de la bi-résidentialité. Rentrer chez soi chaque soir ou une fois par semaine? Rapport final Predit

31. Carrier M, Demaziere C (2012) Introduction La socio-économie des villes petites et moyennes : questions théoriques et implications pour l'aménagement du territoire. Revue d'Économie Régionale et Urbaine $2: 135-149$.

32. Helminen V, Ristimäki M (2007) Relationships between commuting distance, frequency and telework in Finland. Journal of Transport Geography 15 : 331-342.

33. Kaufmann V (2002) Temps et pratiques modales. Le plus court est-il le mieux ? Recherche Transports Sécurité 75 : 131-143.

34. Mariotto M (2013) L'échelon départemental, un atout pour des transports intégrés. Transport Environnement Circulation 219 : 74-79.

35. Drevelle M (2012) Les effets de la mise en service des TCSP urbains sur l'organisation du transport interurbain par autocar : quel impact pour l'accessibilité des territoires d'entre-deux ? Revue Géographique de l'Est 52(1-2).

36. Ollivier-Trigalo M (2009) Politique des transports : où en sont les Régions ? Actions, doctrines et institutionnalisation. Revue d'Economie Régionale et Urbaine 3 : 471-490.

37. SNCF (2008) Ligne Nouvelle Montpellier Perpignan, Pré-études fonctionnelles. Études d'offres, trafics et bilans voyageurs régionaux. Rapport final.

38. Lambert H (2013) Le Schéma de mobilité du Département du Nord : une conception par et pour les Nordistes. Transport Environnement Circulation 219: 70-73.

39. Ballet J-C, Clavel R (2007) Le covoiturage en France et en Europe : État des lieux et perspectives. CERTU, Lyon.

40. Raux C, Crozet Y, Joly I, Ma T-Y, Ovtracht N, Thiebaut V, Kaufmann V, Vincent S, Cornelis E (2010) EuroCities DATTA, Livrable 5 Individual perceptions of travel time and spaces. ANR, Programme blanc 2007.

41. Crozet Y, Joly I (2004) Budget-temps de transport: les sociétés tertiaires confrontées à la gestion paradoxale du bien le plus rare. Les Cahiers Scientifiques du Transport $45: 27-48$.

42. Viry G, Kaufmann V, Widmer D (2009) La grande mobilité géographique pour des raisons professionnelles en suisse : une étape de vie préparentale? Recherches familiales 1(6) : 67-80.

43. Nessi H (2012) Incidences du contexte urbain et du rapport au cadre de vie sur la mobilité de loisir. Thèse de doctorat, Université Paris-Est

44. Vincent-Geslin S, Joly I (2012) Raisons et pratiques de la pendularité intensive. Le temps de trajet, entre temps subi et temps choisi. Les Cahiers scientifiques du transport 61:159-186.

45. Vignal C (2006) Concilier mobilité résidentielle et mobilité professionnelle : cadrage statistique et pistes de réflexion. Horizon stratégiques $2: 37-47$.

46. Ortar N (2005) Le paradoxe de l'ancrage et de la mobilité en zone rurale et périurbaine. In Bonnet L, Bertrand L Mobilités, habitat et identités, INED, Documents de travail. 